\title{
Beobachtungen der Sonnenfinsternis vom 7. April 1921.
}

Auf der Universitätssternwarte Bonn.

Bei Beginn der heutigen Sonnenfinsternis zog hier ein ziemlich dichter Wolkenschleier vor der Sonne vorüber. Ich beobachtete deshalb am $3^{6} \mathrm{~cm}$-Refraktor, der auf $20 \mathrm{~cm}$ äbgeblendet war, das projizierte Sonnenbild und bemerkte den ersten Eingriff um $2 \mathrm{I}^{\mathrm{h}}{ }_{1 \mathrm{O}^{\mathrm{m}}} 7_{7} \mathrm{~s} 8$ Sternzeit Bonn, gleich April 7 I $9^{\mathrm{h}} 37^{\mathrm{m}} 49^{\mathrm{s}} \cdot 3$ mittlere Zeit Greenwich; Beobachtung nach Umständen gut.

Gegen Schluf der Finsternis war der Himmel klar, sodaß mit einem Okular von 240 facher Vergrößerung und grüngelbem Blendglas beobachtet werden konnte. Das Ende notierte ich um $23^{\mathrm{h}} 45^{\mathrm{m}} 29$ :8 Sternz. Bonn, gleich $22^{\mathrm{h}} 12^{\mathrm{m}} 35^{\mathrm{s}} \div 9$ mittlere Zeit Greenwich; $2^{\text {s }}$ vor dieser Zeit sah ich den Eingriff noch deutlich, $3^{\mathrm{s}}$ nach ihr bestimmt nicht mehr. Der Rand war recht wallend.

Herr Dr. Hopmann hat am Sechszöller mit Projektion das Ende um $23^{\mathrm{h}} 45^{\mathrm{m}} 22^{\mathrm{s}} \cdot 3$ Sternz. Bonn, Herr Prof. Monnichmeyer und Cand. König am fünffüßigen Fraunhofer ebenfalls mit Projektion um $23^{\mathrm{h}} 45^{\mathrm{m}} 18^{\mathrm{s}}$ bezw. I $5^{\mathrm{s}}$ beobachtet.

Universitäts-Sternw. Bonn, I 92 I April 8. F. Küstner.

Auf der Universitätssternwarte Kopenhagen.

r. Am $360 \mathrm{~mm}$-Refraktor.

F. Fohannsen April 7 Austritt $22^{\mathrm{h}} 26^{\mathrm{m}} 50^{\mathrm{s}} 4 \mathrm{~m}$. Z. Gr.

$\begin{array}{ll}\text { E. Fuul } & 52.4 \\ \text { A. Nielsen } & 49.4 \\ \text { E. Rönnau, } & 5 \mathrm{I} .9 \\ \text { I. V. Setsoft } & 5 \mathrm{I} .4 \\ \text { E. Strömgren } & 5 \mathrm{I.2} \\ \text { F. M. Vinter-Hansen } & 49.4\end{array}$

2. Am $90 \mathrm{~mm}$-Utzschneider und Fraunhofer-Refraktor.

\begin{tabular}{|c|c|c|c|}
\hline & Eintritt & Austritt & $\begin{array}{c}\text { Durchmesser d } \\
\text { Sonnenbildes }\end{array}$ \\
\hline Braae & $19^{h} 5^{I^{m}} 1 O^{s}$ & $22^{\mathrm{h}} 26^{\mathrm{m}} 5^{\mathrm{I}^{\mathrm{s}}}$ & s $\quad 19 \mathrm{~cm}$ \\
\hline
\end{tabular}

3. Am $85 \mathrm{~mm}$-Zeiß-Refraktor.

Ұ. Fischer-Petersen $\quad 9^{\mathrm{h}} 5^{1^{\mathrm{m}}} \circ 0^{\mathrm{s}} 7 \quad 22^{\mathrm{h}} 26^{\mathrm{m}} 4^{8 \mathrm{~s}} \cdot \quad$ Io $\mathrm{cm}$

Alles Projektionen auf weißem Karton.

Die Zehntel sind in der Hauptsache Rechnungsresultate.

Observatorium Kopenhagen, I 921 April 8. E. Strömgren.
Auf der Landessternwarte Königstuhl-Heidelberg.

Kurz vor Beginn der Finsternis begann sich der Himmel mit Cirrusgewölk zu beziehen, das stellenweise gute Durchblicke gestattete, meist aber die Sonne stark verhüllte und gegen Ende unsichtbar machte. Die Austritte konnten nicht gesehen werden. Von Eintritten wurde beobachtet, nach M. E. Z. :

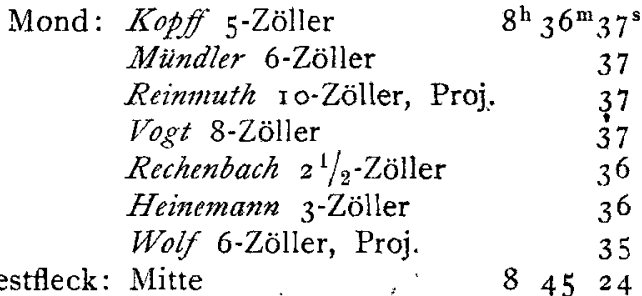

Der der Ostgruppe vorangehende Fleck: Mitte

$93429 \quad(W o)$.

Erster Kern des größeren Flecks: Vorangehender Rand Mitte

Zweiter Kern des größeren Flecks:

Kleiner Fleck: Rand

Mitte

$\begin{array}{rrll} & & 39 & \left(W_{0}\right) \\ & & \\ & 43 & (M u) \\ & 45 & (W o) \\ 34 & 57 & (M u) \\ 35 & 0 & (W o) \\ & 12 & (V o) \\ & & 33 & (M i i) \\ & & 33 & \\ 9 & 35 & 33 & (W o) .\end{array}$

Án 6-Zöller sind i g Phasenbilder aufgenommen worden $(W o)$. Temperaturbeobachtungen verschiedener Art wurden gemacht (Reinm., Heinem.), Himmelshelligkeiten gemessen $\left(V_{o}\right)$ und atmosph. Störungen an der Funkstation fortlaufend beobachtet $(F$. Wolf $)$. Alles durch Wolken wenig oder kaum brauchbar.

Königstuhl, r 92 I April 9.

M. Wolf.

A uf der Universitässternwarte Jena wurden Beobachtungen durch. Regenwetter vereitelt. $O$. Knopf.

Auf der Sternwarte Utrecht.

Erste Berührung $19^{\mathrm{h}} 59^{\mathrm{m}} 42^{\mathrm{s}} \mathrm{m}$. Z. Utrecht; letzte Berührung $22^{\mathrm{h}} 32^{\mathrm{m}} 44^{\mathrm{s}}$. Beobachter van der Bilt am Zehnzöller. Venus wurde bequem mit bloßem Auge aufgefunden.

\section{Neuer Komet 1921 a $($ Reid).}

Der Komet wurde nach den bei der Redaktion uber Kopenhagen eingegangenen telegraphischen Nachrichten der Sternwarte Uccle am I 3 . März I 92 I in Kapstadt von $W$. Reid aufgefunden. Bei der Auffindung hatte er die Heligkeit $9^{\mathrm{m}}$ Eine erste Ortsbestimmung erfolgte in Johannesburg am 14. März, weitere vom I 8. und 25. März wurden aus Algier mitgeteilt. Die dadurch ermöglichte erste Bahnbestimmung stellte die wirkliche Bahn schon ziemlich nahe dar. Eine Verbesserung dieser Bahn wurde von Herrn $M$. Ebell im Anschluß an die Beobachtungen März I 4 Johannesburg, März 25 Algier, April 4 Kopenhagen ausgeführt und ergab die Elemente und Aquatorialkonstanten:

$$
\begin{aligned}
& T=1921 \text { Mai } 10.00992 \mathrm{~m} . Z . G r \text {. } \\
& \omega=64^{\circ} 29^{\prime} 9^{\prime \prime} 7 \\
& \delta=26821 \quad 24.6\} \text { I } 921.0 \\
& i=\mathrm{I} 32 \quad 2 \quad 5^{6.5} \\
& \log q=0.004032
\end{aligned}
$$

Darstellung des mittl. Ortes $B-\mathrm{R}: \mathrm{d} \lambda=+0 .{ }_{4} \mathrm{~d} \beta=-9.7$.

Heliozentrische Äquatorialkoordinaten 1921.0 .

$$
\begin{array}{r}
x=[9.82614] \cdot r \cdot \sin \left(v+2.46^{\circ} 56^{\prime} 31\right) \\
y=[9.98146] \cdot r \cdot \sin (v+31737.67) \\
z=[9.90063] \cdot r \cdot \sin (v+3428.99) \\
H . \text { Kabold. }
\end{array}
$$

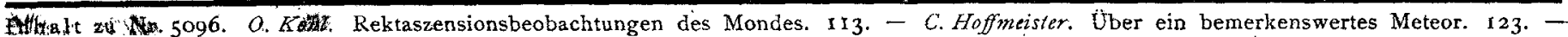
Beobachtungen der Sonnenfinsternis vom 7. April 1921. 127. - Neuer Komet 1921 a (Reid). 127.

Gequlbsen zg2x April t6. Herausgeber: H. Kobold. Druck von C. Schaidt. Expedition: Kiel, Moltkestr, 8o. Postseheck-Konto Nr. 6238 Hamburg 11. 